\title{
Antidepressant-like effect of the saponins part of ethanol extract from SHF
}

Yan Lianga, Xu Yang ${ }^{b}$, Xiaojian Zhang ${ }^{a}$, Hongquan Duan ${ }^{b}$, Meina jin ${ }^{b}$, Yan Sun ${ }^{b}$, Hengjie Yuan, Junqiang Li ${ }^{\text {b }}$ Yuedong Qia*, Wei Qiao ${ }^{\text {b* }^{*}}$

a Department of Pharmacy, The First Affiliated Hospital, Zhengzhou University, Zhengzhou 450000, PR China

b Tianjin Key Laboratory on Technologies Enabling Development of Clinical Therapeutics and Diagnostics, School of Pharmacy, Tianjin Medical University, Tianjin 300070, PR China c Department of Pharmacy/Tianjin Neurological Institute, Tianjin Medical University General Hospital, Tianjin 300052, PR China

E-mail: zdyuedongqi@163.com, qiaowei@tmu.edu.cn

Keywords: Suanzaorenhehuan Formula; saponins part; antidepressant; monoamine oxidase; antioxidative enzyme

\section{ABSTRACT}

Ethnopharmacological relevance: Suanzaorenhehuan Formula (SHF) has been used for treating depression-like disorders for many years in China. The saponins part of the SHF (SSHF) extract was the antidepressant effective component.

Aim of study: To investigate the antidepressant-like effect of SSHF and its possible mechanisms.

Materials and methods: Experimental approaches including the forced swim test (FST), the tail suspension test (TST) and unpredictable chronic mild stress (UCMS) were used to evaluate the effects of SSHF. The possible mechanisms were explored by measuring monoamine neurotransmitter in mice frontal cortex and hippocampus, testing monoamine oxidase enzyme (MAO) activities, antioxidant enzyme activities and free radicals levels in the brains of UCMS-exposed mice.

Results: The results showed that SSHF $(10,20,40 \mathrm{mg} / \mathrm{kg})$ significantly decreased the immobility period in FST and TST in mice after two-week treatment. Whereas, SSHF had no significant effect on locomotor activity in mice. It was also found that the serotonin (5-HT) and noradrenaline (NE) levels in the hippocampus and frontal cortex were significantly increased only in $40 \mathrm{mg} / \mathrm{kg}$ SSHF treated mice. In addition, SSHF $(10,20,40 \mathrm{mg} / \mathrm{kg})$ significantly inhibited monoamine oxidase-A (MAO-A) and monoamine oxidase-B (MAO-B) after 21-day UCMS exposure. SSHF (10, 20, 40 $\mathrm{mg} / \mathrm{kg}$ ) significantly decreased the nitrous oxide (NO) levels, and increased the activities of total antioxidant capability (T-AOC), glutathione peroxidase (GSH-PX), and catalase (CAT) in different degrees in the brains of UCMS-exposed mice.

Conclusions: Our results suggested that SSHF may effectively produce an antidepressant-like effect, which appeared to involve the serotonergic, noradrenergic, monoamine oxidase enzyme and antioxidant systems.

\section{Introduction}

Depression is a common debilitating disorder which can be triggered by chronic psychosocial stress, with well-characterized etiological factors in vulnerable individuals (Wang et al., 2014). According to a report from the World Health Organization (WHO), depression is the fourth highest contributor to the global burden of disease and is predicted to be in second place by 2020 (Peng et al., 2012).Today several kinds of antidepressant drugs have been widely available in the pharmaceutical market, including tricyclic antidepressants(TCAs), selective serotonin reuptake inhibitors(SSRIS), serotonin-noradrenaline reuptake inhibitors(SNRIs), monoamine oxidase inhibitors(MAOIs), noradrenergic and specific serotonergic antidepressants (NaSSAs)(Sultana et 
al., 2014). However, most antidepressant drugs lead to a series of side-effects such as metabolic syndrome, high prolactin, sedation, abnormal liver function (Sagud et al., 2011). Furthermore the efficacy of the pharmacotherapy above-mentioned antidepressant drugs does not exceed 40-50\% and is unsatisfactory (Lasoń et al., 2013). Thus, new drugs with high effectiveness and less adverse effects are still needed.

Suanzaorenhehuan Formula (SHF) was originally recorded in "The Shen Nong Ben Cao Jing", which was written in the Hou-Han Dynasty (A.D.25-220) (Liu et al., 2012). It contains four herbs: Semen Ziziphi spinosae (dried ripe seeds of Rhamnaceae plants Ziziphus jujube Mill. Var. spinosa(Bunge) Hu ex H.F.Chou), Cortex Albiziae(dried stem bark of the leguminous plant Albizzia julibrissin Durazz ), Radix Paeoniae Alba(dried root of Ranunculaceae peony Paeonia lactiflora Pall. ) and Semen Platycladi(dried seeds of Cupressaceae Platycladus orientalis(L.) Franco orientalis). All these four drugs had long been used in various prescriptions to treat depression-like disorders respectively (Sun et al., 2011; Zhu, 1988; Zhang et al., 1998; Xie and Wang, 2005; Shi and Jin, 2011; Sun et al., 2010). In China, SHF has been widely used for mental depression among the populace (Zhang, 2003), especially successfully in clinical treatment at Tianjin Anding Hospital in recent years. In our previous studies, we found that the ethanolic SHF extract $(50,100,200 \mathrm{mg} / \mathrm{kg}$ ) exerted antidepressant-like effects in behavioral despair tests including forced swim test (FST) and tail suspension test (TST) in mice. The effects may be mediated by MAO inhibition and the modulation of neurotransmitter in the brain tissue (Liu et al., 2012). The SHF extract contains two main active fractions, flavonoid glycosides and saponins. And the previous study has preliminarily shown the saponins part of the SHF (SSHF) extract was the antidepressant effective components (Ren et al., 2011). However, the antidepressant-like effect of the SSHF has not been testified so far. The present study was to investigate the antidepressant-like effect of the SSHF through behavioral tests. Besides, the levels of 5-HT, NE, DA, MAO, T-AOC, GSH-PX, CAT and NO in mice brain were also analyzed to find out the probable antidepressant mechanisms of SSHF.

\section{Materials and methods}

\subsection{Preparation of the saponins part of SHF (SSHF)}

Traditional Chinese medicines Semen Ziziphi spinosae, Cortex Albiziae, Radix Paeoniae Alba and Semen Platycladi were purchased from a local herbal medicine market (Tianjin, China). They were identified and authenticated by Professor Ye Zhou (School of Pharmacy, Tianjin Medical University, China) based on their microscopic and macroscopic characteristics. They were mixed at a ratio of 24:13:6:10 and the mixture (270g) was used to prepare SHF powder according to the method described by Liu (Liu et al., 2012). The SHF powder was dissolved in distilled water $(1: 100, w / v)$ and was successively extracted with petroleum ether and $n$-butanol whose fraction was combined, then concentrated to a strong liquor under vacuum at $40^{\circ} \mathrm{C}$, and further washed with $1 \% \mathrm{NaOH}$ to remove the flavones ingredients. The solvent of the $\mathrm{n}$-butanol extract was removed by using a rotary evaporator under low pressure. The yield of the extract was $0.53 \%$ (w/w).

The saponins part of SHF (SSHF) were analyzed by using HPLC fingerprinting analysis. HPLC analysis was performed on an Agilent 1100 series equipped with a DAD. The separation was carried out on an Ameritech AccurasiL C18 column ( $250 \mathrm{~mm} \times 4.6 \mathrm{~mm}$ i.d., $5 \mu \mathrm{m}$ ). The analysis was achieved with gradient elution using (A) acetonitrile and (B) water (containing $0.1 \%$ phosphoric acid) as the mobile phase. The gradient condition was: $0112 \mathrm{~min}, 22 \% \sim 30 \% \mathrm{~A} ; 12 \sim 13 \mathrm{~min}$, 
30\% 33\%A; 13 26min, 33\% 39\%A; 26 31min, 39\% 42\%A; 31 34min, 42\%A; 34 40min, 42\% 63\%A; 40 56min, 63\% 77.8\%A; 56 62 $\mathrm{min}, 77.8 \% \mathrm{~A}$; 62 66min, 77.8\% 78.2\%A; 66 85min, 78.2\% 100\%A, 85 90min, 100\%A。By referring to standards, the chromatographic fingerprinting analysis showed that the main components of SSHF were jujuboside $A$, jujuboside B, Betulinic acid and Betulin (Fig. 1).

\subsection{Reagents}

Serotonin (5-HT) and benzylamine were obtained from J\&K Chemical Co. Ltd. (Beijing, China). 5-Hydroxytryptaphan (5-HTP) was purchased from Fluka AG Chemical Co. (Buchs, Switzerland). Noradrenaline was obtained from National Institutes for Food and Drug Control (Beijing, China). Dopamine was supplied by Tokyo Chemical Industry Co. Ltd. (Tokyo, Japan). Fluoxetine hydrochloride was obtained from Lilly S.A. (Alcobendas, Spain). Venlafaxine was purchased by Wyeth Medica Ireland (Kildare, Ireland). Total anti-oxidant capability (T-AOC), glutathione peroxidase (GSH-Px), catalase (CAT), and nitric oxide (NO) test kits were purchased from NanjingJiancheng Bioengineering Institute (China). All other reagents and solvents were of analytical grade.

\subsection{Animals}

Male ICR mice weighing 18-20g were obtained from the Animal Center of the Institute of Radiation Medicine Chinese Academy of Medical Sciences. The animals were housed in groups, maintained on a standard condition (temperature $22 \pm 2{ }^{\circ} \mathrm{C}$, humidity $50 \pm 10 \%, 12-\mathrm{h}$ light/dark cycle, lights on at7:00 AM), and allowed free access to food and water. The animals were allowed to acclimatize to the laboratory for at least 3 days before experiment. Experiments in this study were carried out between 9:00 AM and 3:00 PM and performed in accordance with NIH Guide for Care and Use of Laboratory Animals.

\subsection{Drug Administration}

Mice were randomly divided into six groups including control and experimental groups $(\mathrm{n}=$ 10/group). Mice in control and vehicle groups were administered with distilled water. Animals in other three groups were respectively treated with SSHF at the doses of 10, 20 and $40 \mathrm{mg} / \mathrm{kg}$. Mice in rest group were administered with fluoxetine $(7.5 \mathrm{mg} / \mathrm{kg})$ or venlafaxine $(9.4 \mathrm{mg} / \mathrm{kg})$. All drugs were orally administered short-term (7 days), sub-chronically (14 days) and chronically (21 days) between 9:00 AM and 2:00 PM. During test days, administration was performed 1h prior to behavioral testing.

\subsection{Forced swim test}

The test was carried out according to the method described by Porsolt et al (Porsolt et al., 1997). Mice were placed individually in glass cylinders (height20 cm, diameter $14 \mathrm{~cm}$ ) filled with $15 \mathrm{~cm}$ high water (maintained at $25 \pm 1^{\circ} \mathrm{C}$ for $6 \mathrm{~min}$ ), and the duration of immobility was recorded during the last $4 \mathrm{~min}$ of the test. The duration of immobility was regarded as the time spent by the mouse floating in the water without struggling and making only those movements necessary to keep its head above the water.

\subsection{Tail suspension test}

The tail suspension test was performed according to previous report (Steru et al., 1985). Briefly, mice were individually suspended from the edge of a rod with a clamp $(1 \mathrm{~cm}$ from the tip of the end $)$ in a box $(25 \mathrm{~cm} \times 25 \mathrm{~cm} \times 30 \mathrm{~cm})$ with the head $5 \mathrm{~cm}$ from the bottom. All tests was conducted in a darkened room with minimal background noise (Gu et al., 2012). A 6-min test session was employed, and the duration of immobility was recorded during the final 4 min 
interval of the test. Mice were considered immobile when they were completely motionless (Yin et al., 2011).

\subsection{Open-field Test}

The locomotor activity was usually assessed in the open field paradigm, as previously described (Liu et al., 2012). The open-field apparatus was made of a circular base $(80 \mathrm{~cm}$ in diameter, $20 \mathrm{~cm}$ high wall) with three concentric circles of 14,28 , and $42 \mathrm{~cm}$ radius, were divided into $36 \mathrm{U}$ without walls. The center was illuminated with a $60 \mathrm{~W}$ electric bulb, hung directly 40 $\mathrm{cm}$ above it. During all the experiments, the laboratory room was dark. Mouse was placed individually into the center of the arena and left free to explore the arena. The apparatus was cleaned and dried between tests. The ambulation (the number of crossing sector lines with all four paws), rearing (number of times mouse stood on its hind limbs) and numbers of grooming were recorded for $3 \mathrm{~min}$ (Liu et al., 2012).

\subsection{5-hydroxytryptophan (5-HTP) induced head twitches test}

To investigate the possible serotonergic mechanism in the antidepressant-like effect of SSHF, 5-HTP induced head-twitch test was performed as described previously (Corne et al., 1963).SSHF $(10,20$ and $40 \mathrm{mg} / \mathrm{kg}$ ) and fluoxetine $(7.5 \mathrm{mg} / \mathrm{kg}$ ) were orally administered $30 \mathrm{~min}$ before intraperitoneal administration of $5-\mathrm{HTP}(100 \mathrm{mg} / \mathrm{kg})$ for 2 weeks. Immediately after the injection, mice were placed into plastic cages and the cumulative number of head-twitches was counted during a $30 \mathrm{~min}$ period by a trained observer who was blind to drug treatment.

\subsection{Monoamine neurotransmitter levels}

Mouse brain monoamine neurotransmitters NE, DA and 5-HT were measured as described previously (Liu et al., 2012). Animals receiving 14-day drug administration were decapitated $24 \mathrm{~h}$ after the behavioral tests. The total hippocampus and frontal cortex were carefully dissected, weighed and stored at $-80^{\circ} \mathrm{C}$ for determinations. The brain tissue was homogenized and the aqueous phase was used to detect the levels of 5-HT, NE and DA. NE and DA were detected at different activation wavelength and fluorescent wavelength (284 and $480 \mathrm{~nm}, 320$ and $369 \mathrm{~nm}$, respectively). The activation and fluorescent wavelengths were 356 and $468 \mathrm{~nm}$ for 5-HT determination. The concentrations of NE, DA and 5-HT were calculated by compared with its respective standard substance and expressed in $\mathrm{ng} / \mathrm{g}$ weight tissue.

\subsection{Unpredictable chronic mild stress (UCMS) procedure}

The UCMS procedure is a variation of the method described by Chhillar and Dhingra (2013). It consists of repeated mild physical and psychological stressors. Mice were subjected to the following 9 stressors in a random order: $12 \mathrm{~h}$ food deprivation, $12 \mathrm{~h}$ damp sawdust, $1 \mathrm{~min}$ nip tail, $12 \mathrm{~h}$ exposure to a empty bottle,12 $\mathrm{h}$ water deprivation, $2 \mathrm{~min}$ force swimming in cold water at $5^{\circ} \mathrm{C}, 2 \mathrm{~min}$ force swimming in hot water at $45^{\circ} \mathrm{C}, 12 \mathrm{~h}$ cage tilt $\left(45^{\circ}\right)$, overnight illumination. Mice were subjected to two of these stressors once a day randomly over a period of 3 weeks, and the same stressor was not applied for 2 consecutive days.

\subsection{Estimation of monoamine oxidase (MAO) activity}

After 21-day UCMS exposure, mice were sacrificed and the brain samples were rapidly frozen $\left(-80^{\circ} \mathrm{C}\right)$. The MAO activity was assessed as the procedure described previously (Liu et al., 2012; Chhillar and Dhingra, 2013). The brain tissue was homogenized and the mitochondrial fraction was obtained and resuspended in buffer solution. MAO activity was assessed spectrophotometrically (Charles and Mc, 1977). MAO-A and MAO-B were measured at wavelength of $280 \mathrm{~nm}$ and $242 \mathrm{~nm}$ respectively. Blank samples were worked subsequently to 
eliminate errors.

\subsection{Brain oxidative stress measurements}

To investigate the influence of SSHF to the 21-day-UCMS mice, the brain oxidative stress was measured. Brain samples were minced and homogenized with ice-cold isotonic physiological saline into $10 \%$ homogenates and centrifuged at $3000 \mathrm{rpm}$ for $10 \mathrm{~min}$ at $4{ }^{\circ} \mathrm{C}$. The supernatants were then subjected to the measurement of T-AOC, GSH-PX, CAT and NO levels, according to the assay kit manual.

\subsection{Statistical analysis}

All results were expressed as mean \pm SEM. The data of all groups were analyzed by a one-way analysis of variance (ANOVA) followed by Dunnett's test in order to detect inter-group differences. $P<0.05$ was considered as statistically significant.

\section{Results}

\subsection{Effect of SSHF on behavioral parameters}

As shown in Fig.2 A and B, the saponins part of SHF (SSHF) decreased the immobility time in FST compared to vehicle control group after 7 or 14 days treatment. Although the reduction of the immobility time was not statistically significant after one-week treatment with SSHF when compared to the vehicle-treated group, it showed the tendency. After two-week treatment, SSHF at the doses of $10,20,40 \mathrm{mg} / \mathrm{kg}$ and venlafaxine at $9.4 \mathrm{mg} / \mathrm{kg}$ were effective in the FST [ $p<0.01, p$ $<0.05, p<0.01, p<0.01$, respectively].

In Fig. $2 C$ and D, the SSHF $(10,20,40 \mathrm{mg} / \mathrm{kg})$ and venlafaxine $(9.4 \mathrm{mg} / \mathrm{kg})$ led to a marked decrease in the immobility periods in the TST after both one-week and two-week treatment compared with the vehicle-treated group [ $p<0.01, p<0.01, p<0.01, p<0.01$, respectively].

Table 1 illustrates that there were no significant changes in crossing, rearing and grooming numbers compared to vehicle group in the OFT after two-week treatment with $\operatorname{SSHF}(10,20$, $40 \mathrm{mg} / \mathrm{kg}$ ) or venlafaxine $(9.4 \mathrm{mg} / \mathrm{kg})$.

Table 1 Effects of SSHF on the crossings, rearings and grooming of mice in the OFT

\begin{tabular}{lllll}
\hline Groups & Dose $(\mathrm{mg} / \mathrm{kg})$ & Number of crossings & Number of rearings & Number of groomings \\
\hline Vehicle & - & $16.1 \pm 1.1$ & $1.1 \pm 0.4$ & $1.1 \pm 0.3$ \\
Venlafaxine & 9.4 & $15.4 \pm 1.8$ & $1.4 \pm 0.5$ & $0.9 \pm 0.3$ \\
SSHF & 10 & $11.43 \pm 1.0$ & $1.1 \pm 0.4$ & $0.9 \pm 0.3$ \\
SSHF & 20 & $18.14 \pm 1.9$ & $1.6 \pm 0.6$ & $0.8 \pm 0.3$ \\
SSHF & 40 & $13.43 \pm 1.2$ & $1.0 \pm 0.4$ & $0.8 \pm 0.3$ \\
\hline
\end{tabular}

Values were presented as means $\pm \operatorname{SEM}(n=10)$, there was no statistical significance between groups.

\subsection{Effect of SSHF on the 5-HTP-induced-head-twitch response}

As shown in Fig.3, SSHF significantly increased the number of head twitches in comparison to the vehicle-treated group [ $<<0.05, p<0.05$, respectively] at dose of 10 or $40 \mathrm{mg} / \mathrm{kg}$ during a 14-day period. Venlafaxine also showed an increasing effect on the number of head twitches $[p<$ 0.05].

\subsection{Effect of SSHF on monoamine neurotransmitter levels in different brain regions of mice}

Results of the content of monoamine neurotransmitter in mice frontal cortex and hippocampus were shown in Fig.4 and Fig.5 respectively. After two-week treatment, the vehicle-treated group revealed a much lower levels of $5-\mathrm{HT}$ and NE in both frontal cortex $[p<$ $0.05, p<0.01$, respectively] and hippocampus [ $p<0.05, p<0.05$, respectively]. However, there 
was no significant differences between vehicle-treated group and control group with respect to the expression level of DA. There was a U-shaped increasing of NE level in frontal cortex, 5-HT and NE levels in hippocampus after two-week treatment with SSHF at doses of 10, 20, $40 \mathrm{mg} / \mathrm{kg}$. The 5-HT and NE levels were significantly increased only after treated with $40 \mathrm{mg} / \mathrm{kg}$ SSHF for 14 days both in frontal cortex and hippocampus [ $p<0.05, p<0.05, p<0.01, p<0.05$, respectively]. Venlafaxine elevated the $5-\mathrm{HT}$ and NE levels but did not affect DA levels both in mice frontal cortex and hippocampus [ $p<0.05, p<0.05, p<0.05, p<0.05$ respectively].

\subsection{Effect of SSHF on brain monoamine oxidase activity}

Compared to the UCMS-free control group, unpredictable chronic mild stresses significantly increased activities of MAO-A and MAO-B in brains. Fig.6 showed that SSHF $(10,20,40 \mathrm{mg} / \mathrm{kg})$ administered for 21 successive days significantly reduced the activity of MAO-A [ $p<0.01, p<0.05$, $p<0.05$, respectively] and MAO-B $[p<0.01, p<0.01, p<0.05$, respectively] activity in stressed mice as compared to the vehicle-treated groups. Fluoxetine also produced a significant reduction on both MAO-A and MAO-B [ $p<0.01, p<0.05$, respectively].

\subsection{Effect of SSHF on lipid peroxidation of brain}

After 21-day-UCMS exposure, Fig.7 showed a significant increase in NO level $[p<0.01]$ and a tendency of decreasing the activities of T-AOC, GSH-PX and CAT in the brains of vehicle-treated group compared with the control group. The administration of SSHF $(10,40 \mathrm{mg} / \mathrm{kg})$ could significantly raise the activity of T-AOC compared with the vehicle group [ $p<0.05, p<0.05$, respectively]. The activity of GSH-PX was significantly increased only in $40 \mathrm{mg} / \mathrm{kg}$ SSHF treated group compared with the vehicle group $[p<0.01]$. When compared with vehicle group, the brain CAT enzyme activities in SSHF groups $(10,20 \mathrm{mg} / \mathrm{kg}$ ) were significantly increased $[p<0.05, p<$ 0.05]. Fluoxetine also showed a significant influence on increasing the activities of T-AOC, GSH-PX and CAT compared to vehicle group [ $p<0.05, p<0.05, p<0.05$, respectively]. In addition, fluoxetine and $\operatorname{SSHF}(10,20,40 \mathrm{mg} / \mathrm{kg})$ significantly reduced the NO levels in mice brains compared with vehicle-treated group $[p<0.01, p<0.05, p<0.05, p<0.01$ respectively].

\section{Discussion}

Depression diagnosed by symptomatic criteria is a complex mood disorder, the heterogeneity of which suggests that multiple and different biological mechanisms may underlie its etiology. The importance of animal models has been proved during recent advances in experimental neuroscience, including modeling of human mood disorders such as depression and anxiety. The FST and TST, behavioral despair tests are helpful for probing the pathological mechanism of depression and for the evaluation of antidepressant drugs (Porsolt et al., 1978). The duration of immobility reflects state of hopelessness which is the cardinal symptom of depression in human (Castagné et al., 2011). In the present study, we observed that SSHF reduced the immobility duration in the FST and TST after 7 or 14 days treatment which is consistent with the results obtained in previous studies on SHF (Liu et al., 2012). In FST, the SSHF $(10,20,40 \mathrm{mg} / \mathrm{kg})$ only showed a tendency to reduce the immobility duration after one-week treatment, while it led to a significant reduction in the immobility period after two-week treatment. The SSHF $(10,20,40 \mathrm{mg} / \mathrm{kg})$ was effective in decreasing immobility time both after one-week and two-week treatment in TST. As the TST is more sensitive to acute antidepressant treatment (Cryan et al., 2005), the antidepressant-like effects of SSHF were observed earlier in the TST than in the FST. One of the major concerns of these models is that drugs may enhance the locomotor activity and give a false positive effect. However, our study demonstrated that the 
anti-immobility effect of SSHF cannot be attributed to any psychostimulant action, since SSHF did not produced alterations in the locomotor activity assessed in the OFT. Taken together with FST and TST, the SSHF did not produce a dose-dependent manner after administration, it exerted better antidepressant effects at doses of 10 and, $40 \mathrm{mg} / \mathrm{kg}$. This U-shaped dose-response curve, in predictive tests of antidepressant activity, is commonly observed in the literature with plant extracts and their isolated compounds (Freitas et al., 2010; Bukhari and Dar, 2013).

It is well known that the dysregulation of central nervous system is associated with the neurotransmitters noradrenaline, serotonin and dopamine which plays an important role in the pathogenesis of depression. Currently, the most efficacious treatment of major depression is considered to involve an increase in serotonin and/or noradrenaline neurotransmission (Ye et al., 2012). Thus, serotonergic, noradrenergic and dopaminergic systems play the major roles in the actions of antidepressants (Sarris, 2011; Kwon et al., 2010). 5-HTP-induced head-twitch test is usually used to investigate the effects of antidepressant drugs on serotonergic function (Ye et al., 2012).In our study, SSHF (10, $40 \mathrm{mg} / \mathrm{kg})$ significantly enhanced 5-HTP-induced-head-twitch response in mice. It suggested the possible involvement of the serotonergic system in the antidepressant activity of SSHF. To detect the brain monoamine level, we focused on two brain regions, the hippocampus and the frontal cortex. These two brain regions are both critically involved in the regulation of emotion, motivation, learning and memory, all of which may be related to the expression of depression (Ye et al., 2015). Our results showed that SSHF (40mg/kg) led to a marked increase of 5-HT and NE levels in both hippocampus and frontal cortex. However, no valuable change in DA was observed in either of the two brain regions. Thus, it suggests that the antidepressant-like effect of SSHF might be mediated by the serotonergic system and noradrenergic system. And the results were similar with the antidepressant effects of SHF (Liu et al., 2012).

To further investigate the antidepressant-like effects of SSHF, UCMS model was developed. As proposed by Willner (Willner et al., 1992), UCMS appeared more suitable for studying the neurobiological basis of depression and the mechanisms of action of antidepressant drugs compared to acute stress models. In our study, UCMS resulted in significant increase in brain MAO-A and MAO-B activities as compared to the CUMS-free control group. MAO is an enzyme that was responsible for metabolic degradation of catecholamines, serotonin, and other endogenous amines in central nervous system. It exists in two similar molecular forms-A and B.MAO-A has substrate preference for serotonin, is the main target for the antidepressant monoamine oxidase inhibitors. MAO-B has substrate preference for phenylethyl amine (Ng et al., 2008).From our results, the activity of MAO-A and MAO-B was significantly inhibited after 21-day-treatment with SSHF at doses of $10,20,40 \mathrm{mg} / \mathrm{kg}$. Then, it may explain the enhanced activity of 5-HT and NE after two-week treatment with SSHF.

Studies have suggested that depression was accompanied by oxidative stress dysregulation, including abnormal total antioxidant capacity, antioxidants, free radicals, oxidative damage and autoimmune response products (Liu et al., 2015). Among a number of the antioxidant enzymes, CAT is the most important cellular antioxidants. It can participate in many physiological metabolic reactions and scavenge many kinds of free radicals produced in the process of cell metabolism. It can degrade hydrogen peroxide into water and yield oxygen. GSH-Px is a selenium-dependent enzyme that is expressed in the human brain, and plays an important role in the detoxification of reactive oxygen and reactive nitrogen species-related oxidative stress (Birgül et al., 2013). NO 
overproduction, a signaling molecule that is synthesized from L-arginine by nitric oxide synthase (NOS), is seen in patients suffering from major depression and plays a major role in the pathogenesis of major depression. Various NOS inhibitors have been found to be potent antidepressant agents (Ashish and Kulkarni, 2011). The results depicted in Fig.7 demonstrated that the treatment with SSHF $(10,20,40 \mathrm{mg} / \mathrm{kg})$ could increase the activity of T-AOC, GSH-PX, and CAT in different degrees, and decreased the NO levels in UCMS mice brain at the same time. It suggested that the antidepressant-like effects of SSHF might be related to its eliminating of the excessive free radicals and anti-lipid peroxidation.

In summary, our study showed that SSHF exhibited antidepressant-like activities in behavioral despair test. These effects were seemed to be associated with the MAO inhibition, the modulation of monoamine neurotransmitter and antioxidative enzymes, and the elimination of excessive free radicals in the brain tissues of mice. In our previous studies, SHF showed antidepressant-like effects and contained jujuboside A and jujuboside B (Liu et al., 2012). Jujuboside A and jujuboside B are the main bioactive compounds of semen Ziziphi Spinosae with sedative activity (Zhang et al., 2013). Betulinic acid has been reported to produce an antidepressant-like effect in TST (Machado et al., 2013). And betulinic acid and betulinic have also shown antidepressant-like effects in our other studies, which has not been reported yet. Since SSHF also contained jujuboside A, jujuboside B, betulinic acid and betulinic, we deduced that these four components were related to the antidepressant effects of SSHF. Further studies are needed to investigate the bioactive compounds involved in the antidepressant-like effects of SSHF.

\section{Acknowledgements}

This work was supported by the Youth Innovation Fund of the First Affiliated Hospital of Zhengzhou University. This study was also supported by the National Sciences Foundation of China (NSFC) (81173530 and 81102447) and Tianjin Research Program of Applied Basic and Cutting-edge Technologies (12JCZDJC25900).

\section{References}

Ashish, D., Kulkarni, S.K., 2011. Nitric oxide and major depression. Nitric Oxide. 24, 125-131.

Birgül, E.C., Huseyin, O., Omer, A., Isil, G.G., Suleyman, D., Rifat, K., 2013. Analysis of manganese superoxide dismutase (MnSOD: Ala-9Val) and glutathione peroxidase (GSH-Px: Pro $197 \mathrm{Leu}$ ) gene polymorphisms in mood disorders. Bosn J Basic Med Sci. 13(2): 109-113.

Bukhari, IA., Dar, A., 2013. Behavioral profile of Hypericum perforatum (St. John's Wort) extract. A comparison with standard antidepressants in animal models of depression. Eur Rev Med Pharmacol Sci. 17(8) 1082-9.

Castagné, V., Moser, P., Roux, S., Porsolt, R.D., 2011. Rodent models of depression: forced swim and tail suspension behavioral despair tests in rats and mice. Curr Protoc Neurosci. 11-18.

Charles, M., McEwan, J., 1977. MAO activity in rabbit serum, in: Tabor H., Tabor C.W. (Eds), Methods in Enzymology, XVIIB, Academic Press, New York and London. 692-698.

Chhillar, R., Dhingra, D., 2013. Antidepressant-like activity of gallic acid in mice subjected to unpredictable chronic mild stress. Fundam Clin Pharmacol. 27, 409-418.

Cryan, J.F., Mombereau, C., Vassout, A., 2005. The tail suspension test as a model to assessing antidepressant activity: review of pharmacological and genetic studies in mice. Neurosci Biobehav Rev. 29, 571-625.

Corne, S.J., Pickering, R.W., Warner, B.T., 1963. A method for assessing the effects of drugs on the 
central actions of 5-hydroxytryptamine. Br J Pharmacol Chemother. 20, 106-120.

Freitas, A.E., Budni, J., Lobato, K.R., Binfaré, R.W., Machado, D.G., Jacinto, J., Veronezi, P.O., Pizzolatti, M.G., Rodrigues, A.L., 2010. Antidepressant-like action of the ethanolic extract from Tabebuia avellanedae in mice: Evidence for the involvement of the monoaminergic system. Prog Neuropsychopharmacol Biol Psychiatry. 34, 335-343.

Gu, L., Liu, Y.J., Wang, Y.B., Yi, L.T., 2012. Role for monoaminergic systems in the antidepressant-like effect of ethanol extracts from Hemerocallis citrine. J Ethnopharmacol. 139, 780-787.

Kwon, S., Lee, B., Kim, M., Lee, H., Park, H.J., Hahm, D.H., 2010. Antidepressant-like effect of the methanolic extract from Bupleurumfalcatum in the tail suspension test. Prog Neuropsychopharmacol Biol Psychiatry. 34(2): 265-270.

Lasoń, W., Budziszewska, B., Basta-Kaim, A., Kubera, M., Maes, M., 2013. New trends in the neurobiology and pharmacology of affective disorders. Pharmacol Rep. 65, 1441-1450.

Liu, J., Qiao, W., Yang, Y., Ren, L., Sun, Y., Wang, S.,2012. Antidepressant-like effect of the ethanolic extract from Suanzaorenhehuan Formula in mice models of depression. J Ethnopharmacol. 141, 257-264.

Liu, T., Zhong, S.M., Liao, X.X., Chen, J., He, T.T., Lai, S.K., Jia, Y.B., 2015. A Meta-Analysis of Oxidative Stress Markers in Depression. PLoS One. 10(10):e0138904.

Machado, D.G., Cunha, M.P., Neis, V.B., Balen, G.O., Colla, A., Bettio, L.E., Oliveira, A., Pazini, F.L., Dalmarco, J.B., Simionatto, E.L., Pizzolatti, M.G., Rodrigues, A.L., 2013. Antidepressant-like effects of fractions, essential oil, carnosol and betulinic acid isolated from Rosmarinus officinalis L. Food Chem. 136, 999-1005.

Ng, F., Berk, M., Dean, O., Bush, A.I., 2008. Oxidative stress in psychiatric disorders: evidence base and therapeutic implications. Int J Neuropsychopharmacol. 11(6): 851-876.

Peng, Y.L., Liu, Y.N., Liu, L., Wang, X., Jiang, C.L., Wang, Y.X., 2012. Inducible nitric oxide synthase is involved in the modulation of depressive behaviors induced by unpredictable chronic mild stress. J Neuroinflammation. 9:75.

Porsolt, R.D., Bertin, A., Jalfre, M., 1978. Behavioural despair in rats and mice: strain differences and the effects of imipramine. Eur J Pharmacol. 51(3): 291-294.

Porsolt, R.D., Bertin, A., Jalfre, M., 1997. Behavioral despair in mice: a primary screening test for antidepressants. Arch Int Pharmacodyn Ther. 229(2): 327-336.

Ren, L.Y., Qiao, W., Liu, J.S., Yang, Y., W, S.X., 2011. Studies on Antidepressant Effective Fraction of Suanzaoren Hehuan Formula. Traditional Chinese Drug Research \& Clinical Pharmacology. 22, 602-605.

Sarris, J., Panossian, A., Schweitzer, I., Stough, C., Scholey, A., 2011. Herbal medicine for depression, anxiety and insomnia: a review of psychopharmacology and clinical evidence. Eur Neurpsychopharmacol. 21(12): 841-60.

Shi, X.M., Jin, Z.P., 2011. Clinical Applications of Platycladi Seed. Journal of China Traditional Chinese Medicine Information. 03(6), 78-79.

Sagud, M., Mihaljevic-Peles, A., Begic, D., Vuksan-Cusa, B., Kramaric, M., Zivkovic, M., Zivković, M., Jakovljević, M., 2011. Antipsychotics as antidepressants: what is the mechanism? Psychiatr Danub. 23(3):302-307.

Sultana, J., Italiano, D., Spina, E., Cricelli, C., Lapi, F., Pecchioli, S., Gambassi, G., Trifirò, G., 2014. Changes in the prescribing pattern of antidepressant drugs in elderly patients: an Italian, 
nationwide, population-based study. Eur J Clin Pharmacol. 70(4): 469-478.

Steru, L., Chermat, R., Thierry, B., Simon, P., 1985. The tail suspension test: a new method for screening antidepressants in mice. Psychopharmacology (Berl). 85(3), 367-370.

Sun, Y.F., Liang, Z.S., Shan, C.J., Viernstein, H., Unger, F., 2011. Food Chem. 124, 1612.

Sun, F.J., Cheng, H.H., Wang, C.F., Yan, X.S., 2010. Effect of Semen Platycladi Saponins and Semen Platycladi Oil on Improvement of Sleep. World Journal of Integrated Traditional and Western Medicine. 05 (05), 394-395.

Wang, M., Chen, Q., Li, M., Zhou, W., Ma, T.F., Wang, Y., Gu, S.L., 2014. Alarin-induced antidepressant-like effects and their relationship with hypothalamus-pituitary-adrenal axis activity and brain derived neurotrophic factor levels in mice. Peptides.56, 163-72.

Willner, P., Muscat, R., and Papp, M., 1992. Chronic mild stress-induced anhedonia: a realistic animal model of depression. Neurosci Biobehav Rev. 16, 525-534.

Xie, Z.L., Wang, X.H., 2005. Clinical study of Jiawei Sini Decoction in the treatment of 38 dysthymic patients. Chinese Journal of Information on Traditional Chinese Medicine. 12, 8-9.

Ye, L., Hu, Z.P., Du, G.Y., Zhang, J.Z., Dong, Q.J., Fu, F.H., Tian, J.W., 2012. Antidepressant-like effects of the extract from Cimicifuga foetida L. J Ethnopharmacol. 144 (3): 683-91.

Ye, M., Yang, T.L., Qing, P., Lei, X., Qiu, J., Liu, G.Y., 2015. Changes of Functional Brain Networks in Major Depressive Disorder: A Graph Theoretical Analysis of Resting-State fMRI. PLoS One. 10(9):e0133775.

Yin, C., Gou, L., Liu, Y., Yin, X., Zhang, L., Jia, G., Zhuang, X., 2011. Antidepressant-like Effects of L-theanine in the Forced Swim and Tail Suspension Tests in Mice. Phytother Res. 25, 1636-1639.

Zhang, M.Z., Zhang, Q.Y., Cui, G.B., 1998. Clinical study of XiaoYao-San in the treatment of depressive neurosis. Journal of Shangdong University of Traditional Chinese Medicine 22, 34-37.

Zhang, Y., 2003. A mental-tranquilizer-Albizzia julibrssin. Specialized Households 3, 19-20.

Zhang, Y.Q., Zhang, Y., Zhang, K.S., Ma, G.J., Zhang, M.C., Xie, J.B., 2013. Degradation Kinetics of Jujuboside B by Rat Intestinal Flora In vitro with an RRLC-MS-MS Method. J Chromatogr Sci, 52(7): 691-6.

Zhu, Y.P., 1998. Chinese Materia Medica Chemistry, Pharmacology and Applications. Harwood Academic Publishers, The Netherlands, pp. 519-520.

Figure legeds

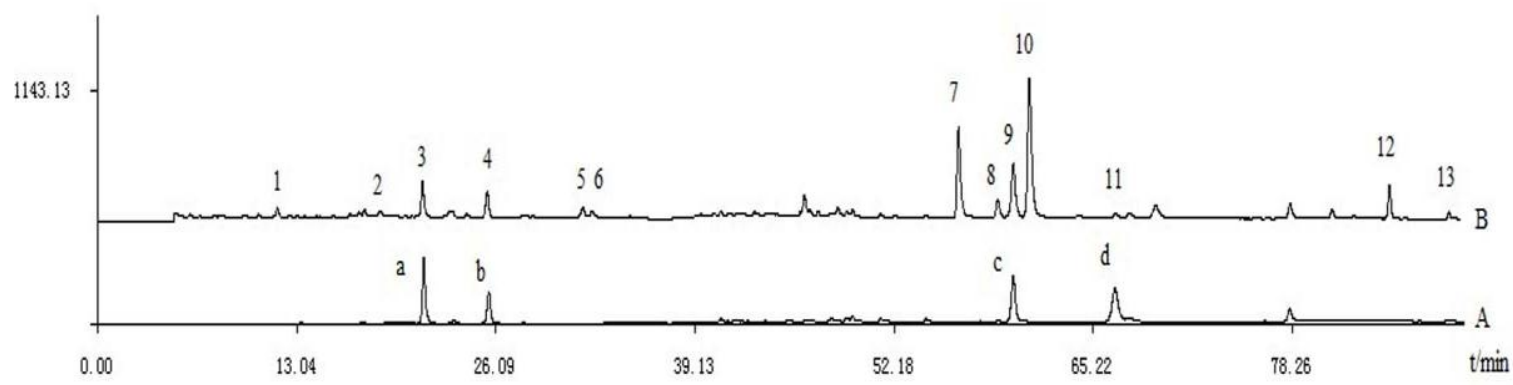

Fig.1. Chromatographic of the saponins part of SHF (SSHF) (B) and standards (A) analyzed by HPLC-DAD technique. (A): a jujubosides $A, b$ jujubosides $B, c$ betulinic acid, $d$ Betulin. (B): 3 jujubosides $A, 4$ jujubosides $B, 9$ betulinic acid, 11 Betulin. 

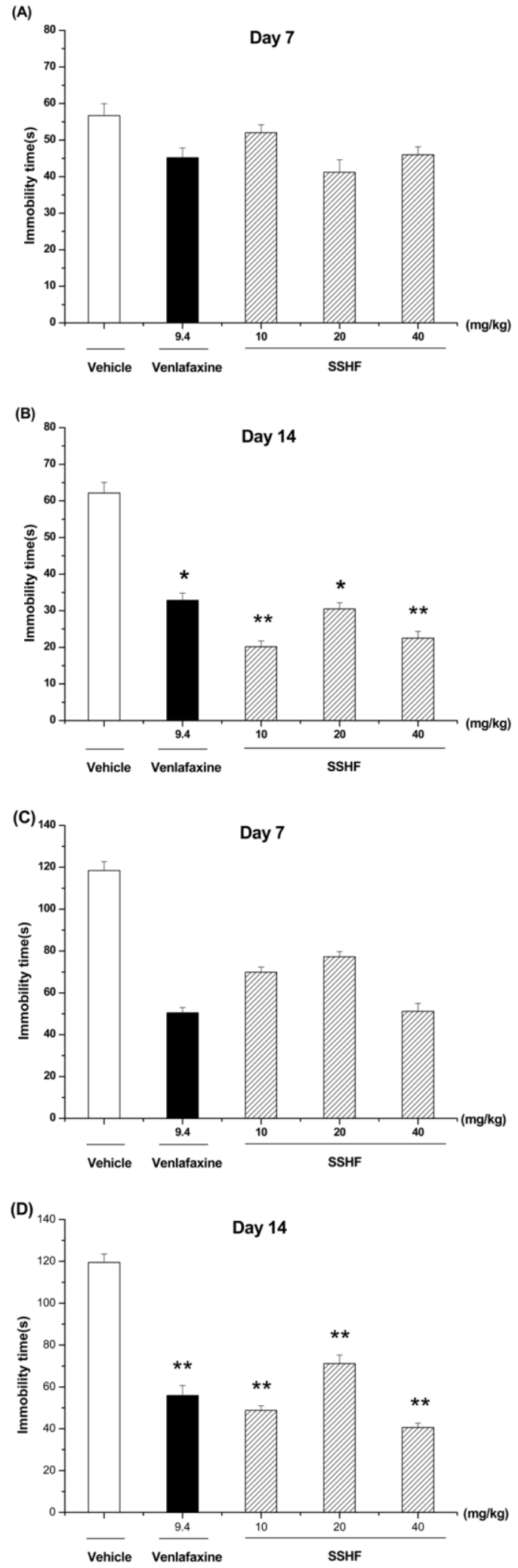

Fig.2. Effects of SSHF and venlafaxine on the immobility time both in FST (A, B) and TST(C, D). The immobility time was measured in mice receiving vehicle, SSHF (10, 20 and $40 \mathrm{mg} / \mathrm{kg})$, venlafaxine 
$(9.4 \mathrm{mg} / \mathrm{kg}$ ) for 7 days (Day 7 ) and 14 days (Day 14). Values were presented as mean \pm S.E.M. $(\mathrm{n}=$ 10). ${ }^{*} p<0.05$ and ${ }^{* *} p<0.01$ compared with the vehicle-treated group.

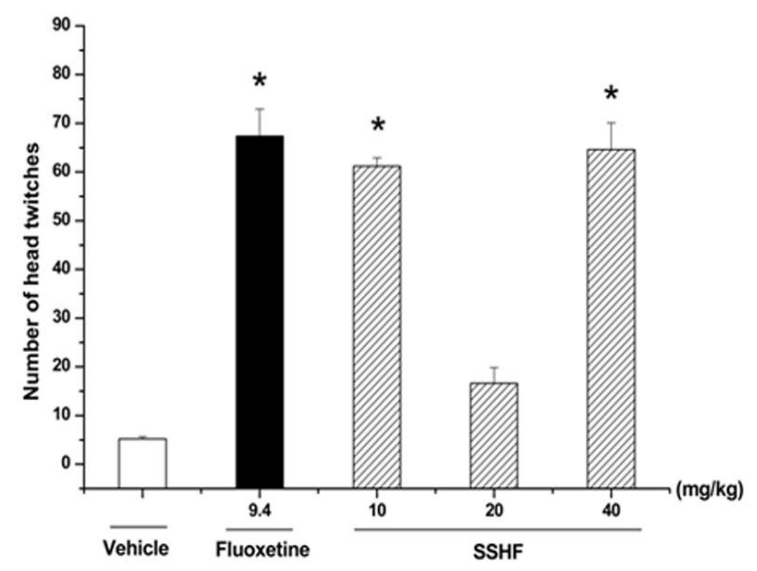

Fig.3 Effects of SSHF on number of 5-HTP-induced head twitches in mice after a two-week treatment. Values were presented as mean \pm S.E.M. $(n=10) .{ }^{*} p<0.05$ and ${ }^{* *} p<0.01$ compared with the vehicle-treated group. 

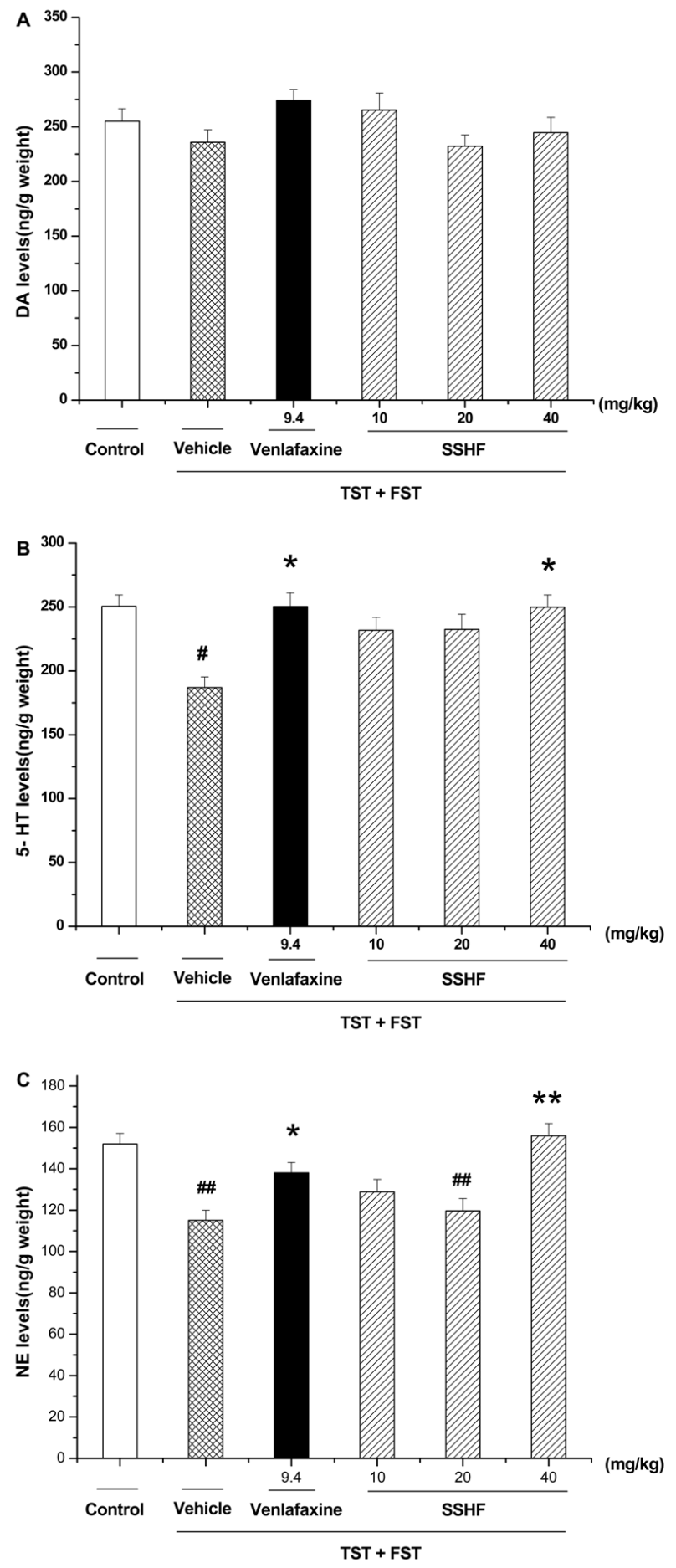

Fig.4 Effects of SSHF on the monoamine neurotransmitter levels in the frontal cortex after TST and FST. SSHF $(10,20,40 \mathrm{mg} / \mathrm{kg})$ and Venlafaxine $(9.4 \mathrm{mg} / \mathrm{kg})$ were given daily for two weeks. Data were expressed as mean \pm S.E.M. $(n=10) . \# p<0.05$ and ${ }^{\#} p<0.01$ compared with the control group. ${ }^{*} p<0.05$ and ${ }^{* *} p<0.01$ compared with the vehicle-treated (TST + FST) group. 

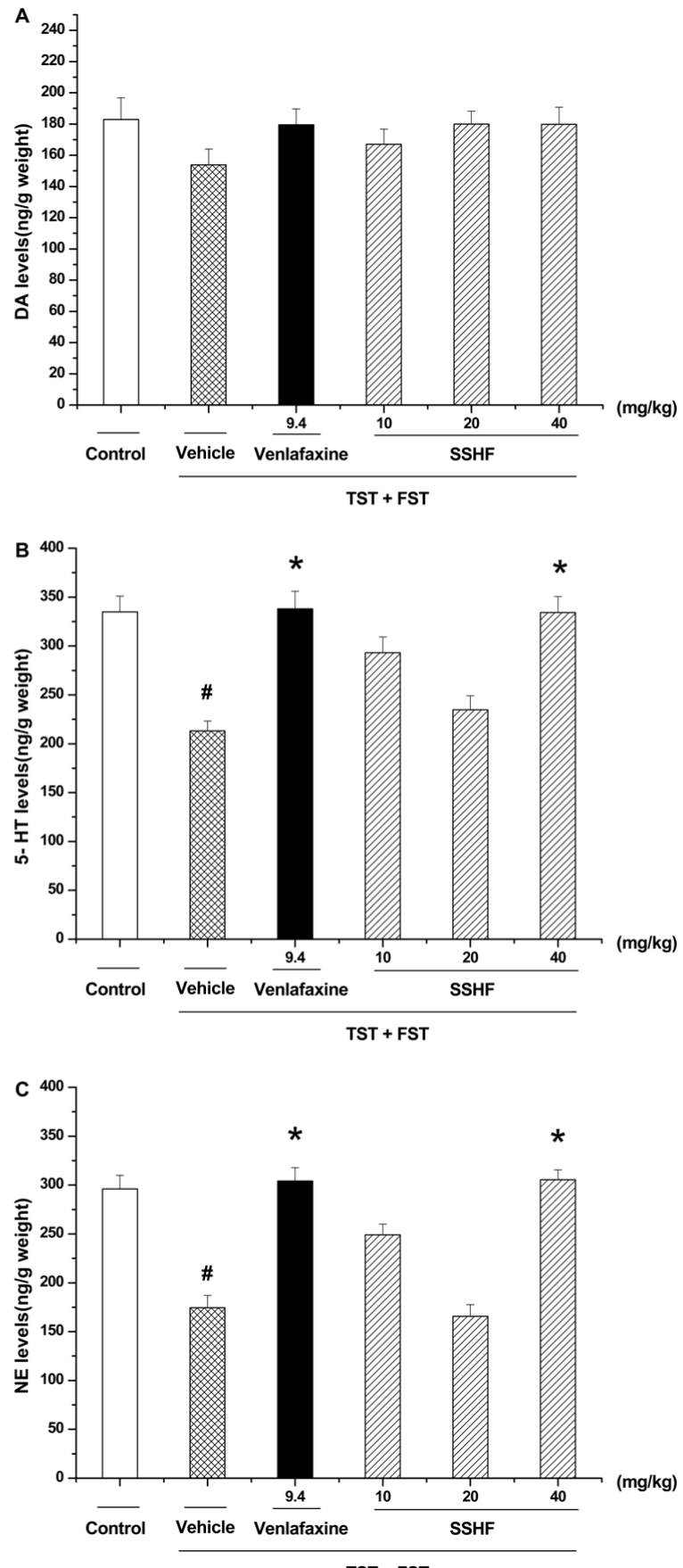

Fig.5 Effects of SSHF on the monoamine neurotransmitter levels in the hippocampus after TST and FST. SSHF $(10,20,40 \mathrm{mg} / \mathrm{kg})$ and Venlafaxine $(9.4 \mathrm{mg} / \mathrm{kg})$ were given daily for two weeks. Data were expressed as mean \pm S.E.M. $(n=10) . \# p<0.05$ and ${ }^{\#} p<0.01$ compared with the control group. ${ }^{*} p<0.05$ and ${ }^{*} p<0.01$ compared with the vehicle-treated (TST + FST) group. 


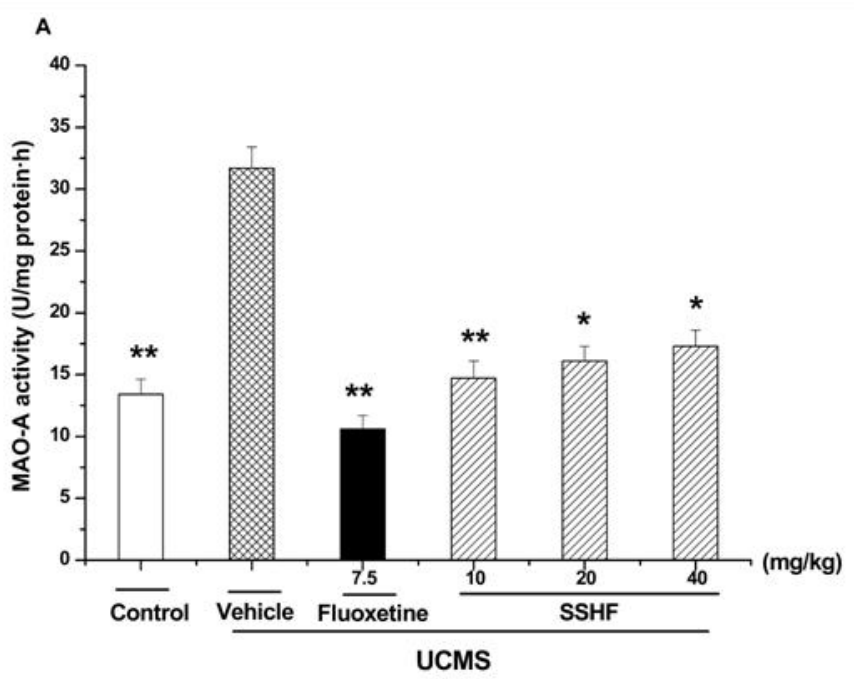

B

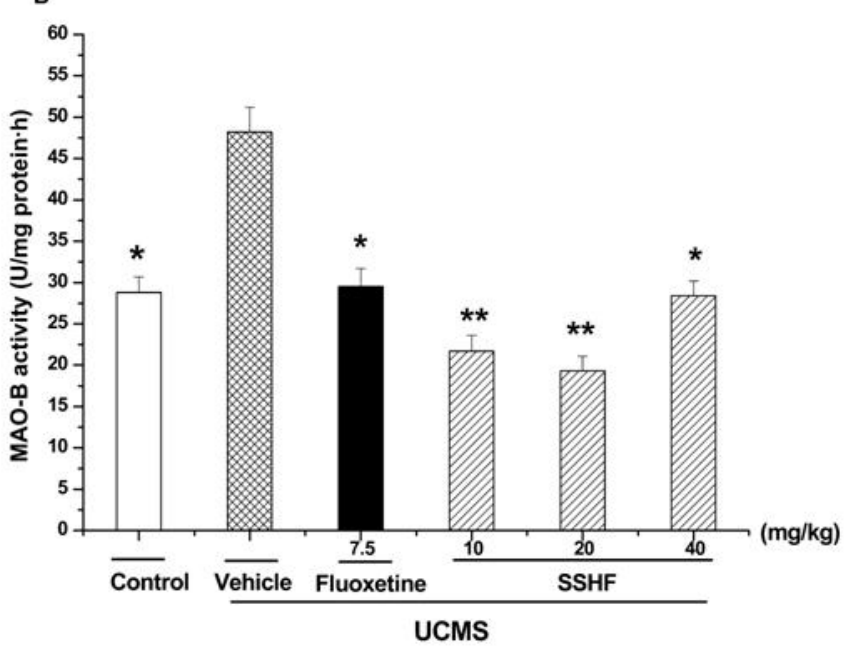

Fig. 6. Effects of SSHF $(10,20$ and $40 \mathrm{mg} / \mathrm{kg})$ and fluoxetine $(7.5 \mathrm{mg} / \mathrm{kg})$ on brain MAO-A and MAO-B activity after 21-day UCMS exposure. Values were expressed as mean \pm S.E.M. $(n=10) .{ }^{*} p$ $<0.05$ and ${ }^{* *} p<0.01$ as compared with the vehicle-treated (UCMS) group. 
A
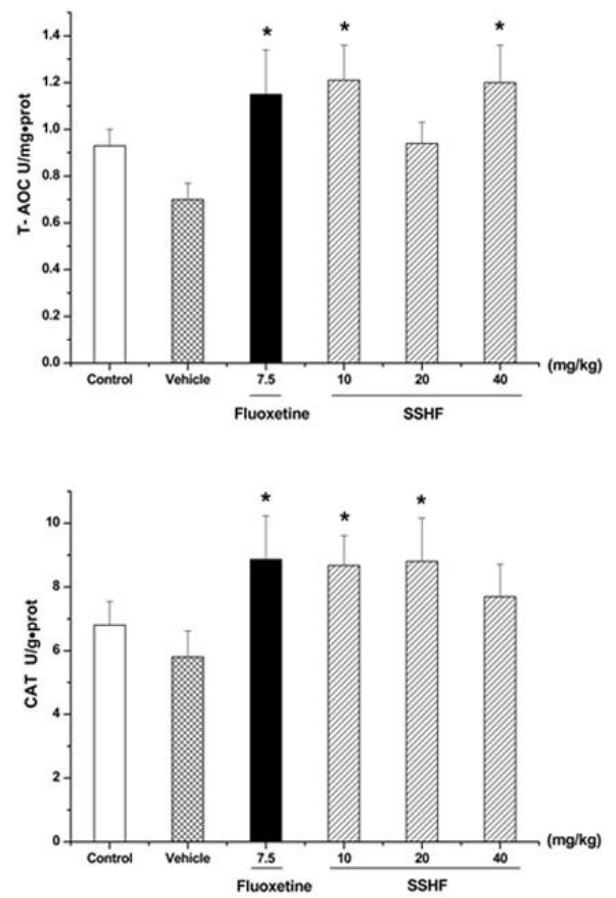

B

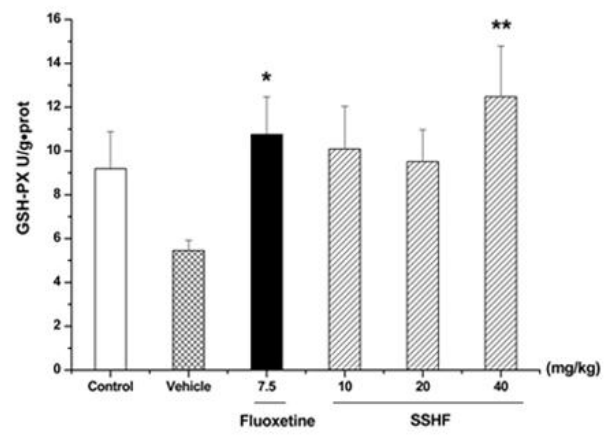

D

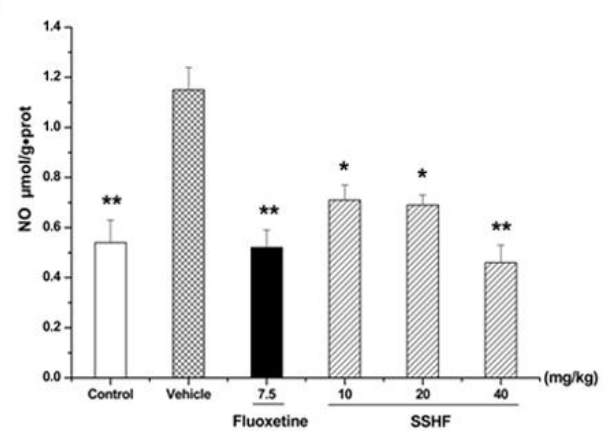

Fig.7 Effects of SSHF (10, 20 and $40 \mathrm{mg} / \mathrm{kg}$ ) and fluoxetine $(7.5 \mathrm{mg} / \mathrm{kg})$ on the activity of T-AOC, GSH-PX, CAT and the NO level of mice brains after 21-day UCMS exposure. Values were expressed as mean \pm S.E.M. $(n=10) .{ }^{*} p<0.05$ and ${ }^{* *} p<0.01$ as compared with the vehicle-treated (UCMS) group. 


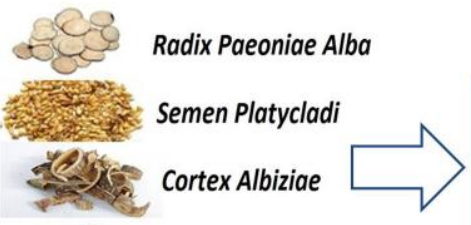

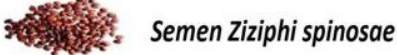

Suanzaorenhehuan Formula (SHF)
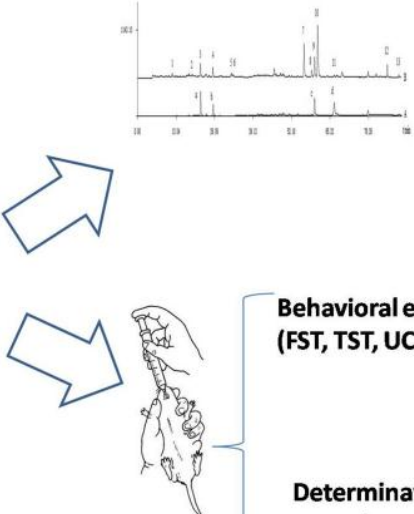

ehavioral experiment

(FST, TST, UCMS)

the SHF

(SSHF)
Determination of monoamine neurotransmitter, monoamine oxidase enzyme and antioxidative enzyme 